\title{
Identification of Artificial Neural Network Models for Three-Dimensional Simulation of a Vibration-Acoustic Dynamic System
}

\author{
Robson S. Magalhães, Cristiano H. O. Fontes, Luiz A. L. de Almeida, Marcelo Embiruçu \\ Programa de Pós-Graduação em Engenharia Industrial, Escola Politécnica, Universidade Federal da Bahia, \\ Salvador, Brasil \\ Email: robsonmagalhaes@ufba.br, cfontes@ufba.br, lalberto@ufba.br,embirucu@ufba.br
}

Received December 18, 2012; revised January 23, 2013; accepted January 30, 2013

\begin{abstract}
Industrial noise can be successfully mitigated with the combined use of passive and Active Noise Control (ANC) strategies. In a noisy area, a practical solution for noise attenuation may include both the use of baffles and ANC. When the operator is required to stay in movement in a delimited spatial area, conventional ANC is usually not able to adequately cancel the noise over the whole area. New control strategies need to be devised to achieve acceptable spatial coverage. A three-dimensional actuator model is proposed in this paper. Active Noise Control (ANC) usually requires a feedback noise measurement for the proper response of the loop controller. In some situations, especially where the real-time tridimensional positioning of a feedback transducer is unfeasible, the availability of a 3D precise noise level estimator is indispensable. In our previous works [1,2], using a vibrating signal of the primary source of noise as an input reference for spatial noise level prediction proved to be a very good choice. Another interesting aspect observed in those previous works was the need for a variable-structure linear model, which is equivalent to a sort of a nonlinear model, with unknown analytical equivalence until now. To overcome this in this paper we propose a model structure based on an Artificial Neural Network (ANN) as a nonlinear black-box model to capture the dynamic nonlinear behaveior of the investigated process. This can be used in a future closed loop noise cancelling strategy. We devise an ANN architecture and a corresponding training methodology to cope with the problem, and a MISO (Multi-Input Single-Output) model structure is used in the identification of the system dynamics. A metric is established to compare the obtained results with other works elsewhere. The results show that the obtained model is consistent and it adequately describes the main dynamics of the studied phenomenon, showing that the MISO approach using an ANN is appropriate for the simulation of the investigated process. A clear conclusion is reached highlighting the promising results obtained using this kind of modeling for ANC.
\end{abstract}

Keywords: Neural Networks; Nonlinear Identification; Dynamic Models; Distributed Parameter Systems; Vibrate-Acoustic Systems

\section{Introduction}

Considering performance requirements, requested in many current applications that use mathematical models, the behavior of the most physical phenomena can be represented by linear systems. The procedures for parametric identification for linear systems are well established and show many theoretical and practical results $[3,4]$. Some systems fail to have their behavior well described by linear models if their frontiers or ranges of values where they are excited are extended. In these cases, it is necessary to use a nonlinear model, and the identification of nonlinear systems using neural networks has been attracting interest and it has been applied successfully elsewhere [5-7].
Modeling techniques which use Artificial Neural Networks (ANNs) have been widely investigated and successfully applied to identification and control problems over the last twenty years. Specifically in vibro-acoustic systems, ANNs have been used in speech recognition [8], in the quality of the sound evaluation in urban areas [9], in the identification of geometric shapes through the identification of natural frequencies in an acoustic response [10] and in the diagnosis of faults [11]. Their great advantages are to work as a "black box" and also to have the ability to approach complex nonlinear mappings, adapting to nonlinearities that exist in behavior patterns (already known) of a system. This nonlinear mapping, which is performed by ANNs is based on the measures of input and output of the process that is going to be mod- 
eled. Because of these characteristics, ANNs are being quite well exploited in the identification of nonlinear dynamic systems currently [12], modeling this nonlinear input/output relationship of the identified process, with the variables changing over time. In these cases, the representation of the dynamics can be characterized through the supplying of the signals set (input and output of the process), which are backward in time at the entrance of the ANN, to include in the modeling the dead time, the memory of the input and the feedback that are associated with the phenomenology of the system, resulting in an input/output representation according to a recurrent architecture [13,14].

ANN is also being used for applications in Active Noise Control (ANC). Bambang [15] developed an application in ANC using recurrent neural networks, where the author presents a learning algorithm for recurrent neural networks based on the Kalman filter. The overall structure for the proposed ANC was formulated using two recurrent neural networks: the first neural network is used to model the secondary source of the ANC, while the second network is used to generate the control signal. Chang [16] proposed a structure based on neural networks in a filtered LMS algorithm, or NFXLMS (Neuralbased Filtered-X Least-Mean-Square algorithm), which is associated with a method to prevent the premature saturation of the backpropagation training algorithm using a best adjustment rate. Zhang [17] studied an ANC system with nonlinearities and proposed unconventional structures of neural networks for modeling the nonlinearity of the acoustic propagation of the primary source in the system. Bouchard [18] introduced a LMS-based algorithm to devise several neural network controllers. The main evaluation criterion used was computation time, aiming at the application of the algorithm in multichan- nel ANC systems.

We present in this paper a methodology for building an ANN model to estimate the noise level in a certain spatial region subjected to noise emissions from a single vibrating source. The proposed model is designed to run in real-time, providing noise level estimation to be used by an ANC control system.

The neural network is trained to estimate the noise level at any point in the contained space in our acoustic system and uses as variables of input of the spatial coordinates of that point and the vibration signal, which is measured at the primary source.

The objective function used in network training is the sum of square errors (difference between the measured value for the noise level and the value predicted by the model in each spatial point). A set of experimental data is chosen for training, using the least squares metric and the obtained neural network is validated through simulations, comparing predictions with another set of data obtained from our experimental platform.

\section{Materials and Methods}

\subsection{Experimental Apparatus and Methodology for Data Collection}

The vibro-acoustic system under study is composed of a centrifugal pump installed in a room (Figure 1(a)). The centrifugal pump is driven by a simple single-phase induction motor and this set is assumed to be our primary source of noise. In this experimental set-up two sensors are used: a fixed ICP (Integrated Circuit Piezoelectric) accelerometer which measures the vibrating signal generated by the primary source and a mobile microphone that measures the sound noise level inside the room, at each point of a previously defined mesh. Figure 2 shows

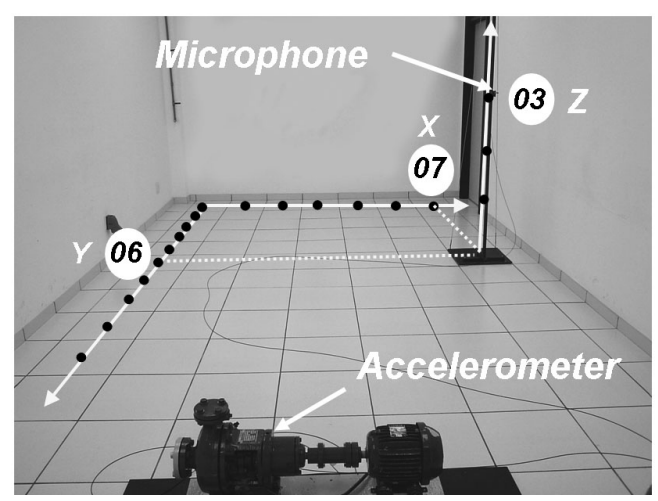

(a)

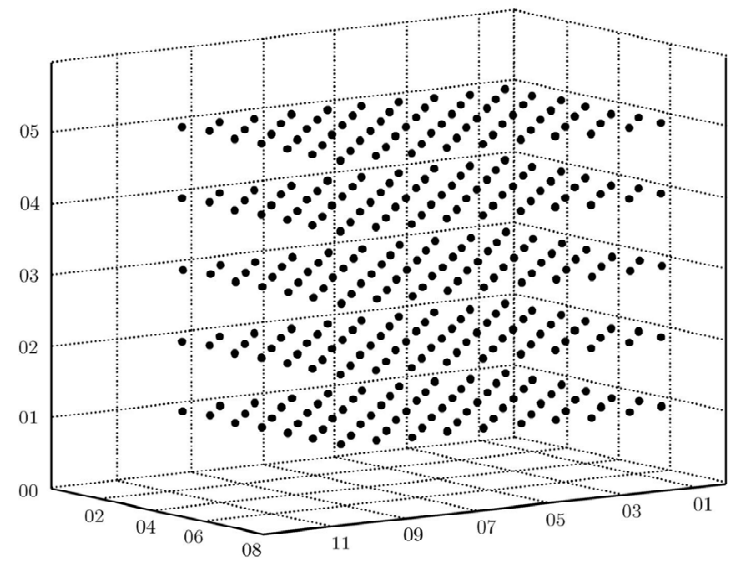

(b)

Figure 1. Acoustic field mapping generated by a rotating machine operating in a closed room identified by coordinates $X, Y, Z: X=1,2, \cdots, 7 \times(0.44 \mathrm{~m}) ; Y=1,2, \cdots, 10 \times(0.44 \mathrm{~m}) ; \quad$ and $Z=1,2, \cdots, 5 \times(0.44 \mathrm{~m})$. Microphone displacement (passive sensor): (a) Experimental setup; (b) Mesh of the 350 collected data $(7 \times 10 \times 5$ positions assumed by the passive sensor). 


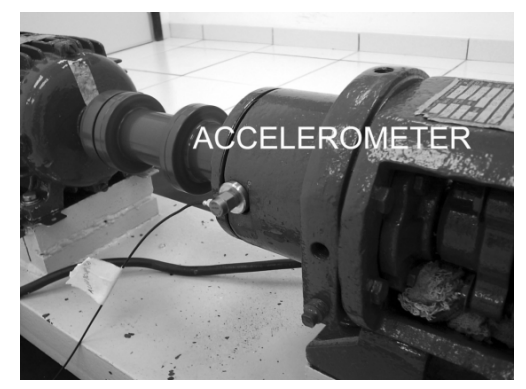

(a)

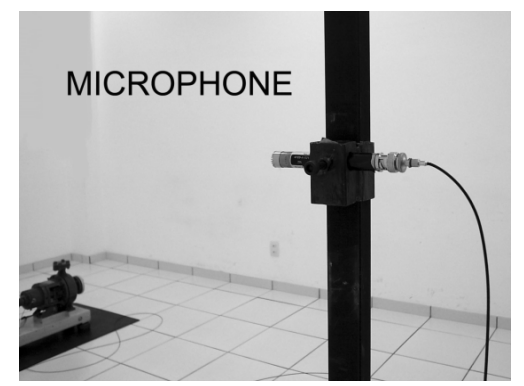

(b)

Figure 2. Experimental apparatus: (a) System input, primary source sensor: pump accelerometer (100mV/g) signal; (b) System output, passive sensor: microphone $(50 \mathrm{mV} / \mathrm{Pa})$ signal.

details of both the accelerometer and the microphone installation. Considering the data collected by the accelerometer installed in the pump, and varying the position of a microphone per 350 predetermined points in the room (they are identified by its coordinates

$X, Y, Z: X=1,2, \cdots, 7 \times(0.44 \mathrm{~m}) ; Y=1,2, \cdots, 10 \times(0.44 \mathrm{~m})$; and $Z=1,2, \cdots, 5 \times(0.44 \mathrm{~m})), 350$ pattern pairs were collected $\left\{\boldsymbol{u}^{p}, \boldsymbol{y}^{p}\right\}$ which represent the dynamic of the vibro-acoustic transmission between the input signal that comes from the accelerometer $(u)$ and the output signal, which comes from the microphone $(y)$. The collected set of pairs $\left(\left\{\boldsymbol{u}^{p}, \boldsymbol{y}^{p}\right\}, p=1,2, \cdots, 350\right)$ defines the group of standard that will be used to train a recurrent ANN network that can best describe the dynamic of the vibroacoustic transmission in the proposed experimental platform.

\subsection{Model Structure: Characteristics of the Used Neural Network}

Since Rosenblatt [19], ANNs have been used to model and simulate nonlinear systems of diverse nature in digital computers or in hardware boards. According to Masson [20], and expressed in Figure 3(a), the topology of an artificial neural network can be expressed through a directed graph characterized by a set of vertices, a set of directed arcs and a set of weights to these $\operatorname{arcs}(W)$. Each vertex in the graph represents a processing unit. A processing unit has $u_{1}, u_{2}, \cdots, u_{R}$ inputs. Based on these inputs and the set of synaptic weights, $W_{1,1}, \cdots, W_{S, R}$, the neurons are evaluated, generally through an activation function applied to a weighted sum of inputs using the synaptic weights as weighting factors [20]. A network with a single layer having $S$ neurons with an arbitrary activation function and with $R$ inputs is shown in detail in Figure 3, which also illustrates its processing unit (neuron). Neural networks frequently have one or more hidden layers of sigmoid neurons (for example, tansig or logsig) [21-23] following by an output layer of linear neurons. If a two-layer network is considered, we have:
- one hidden layer with $S_{\mathrm{I}}$ sigmoid neurons with biases $\boldsymbol{b}^{1}$ which are associated with each neuron;

- one layer with $S_{L}$ output neurons activated by a linear function with biases $\boldsymbol{b}^{2}$, which are associated to each neuron.

By using a sufficient number of neurons $\left(S_{\mathrm{I}}\right)$ in the hidden layer of a two-layer network it is possible to approximate any function with a finite number of discontinuities within an accuracy that is arbitrarily specified [24,25]. This structure is shown in Figure $\mathbf{4}$ and it can be used as a universal approximator of functions. The representation of the system dynamics was characterized through the use of the set of input and output of the process $(u$ and $y$ ), backward in time, placed in the ANN's input, as mentioned previously $[13,14]$ and shown in Figure 5, which also illustrates the internal structure of the adopted ANN (an intermediate layer and an output layer). In this figure $u(n)$ is a sampling of the input signal at time $n, y(n)$ is the sampling of the output signal at time $n, d$ is the delay (dead time) of the system output, relating to the input $u$ and $q$ is the forward shift operator. The scheme shown in Figure 4 (general approximator of functions) is used, therefore this ANN is able in principle to approximate any function with a finite number of discontinuities using a sufficient number of neurons $\left(S_{I}\right)$ in the hidden layer.

The chosen orders for $u$ and $y$ in Figure 5 are the same as those adopted in the work of Magalhaes [1] that presents an extensive discussion about the dynamic behavior expected for the acoustic problem studied. Thus, considering that the $X, Y$ and $Z$ coordinates of any point in a room are also inputs to the net work (in this case static and therefore of zero order), we can conclude that the dynamic ANN to be configured to estimate the $\hat{y}^{p}(n)$ acoustic pressure in an arbitrary spatial position has eight entries. For the time delay the same procedure of Magalhaes [1] is adopted, where $d$ is calculated on a theoretical basis, using sound velocity $(v)$ and longitudenal distance $(Y)$ between primary source and the grid measurement point. 


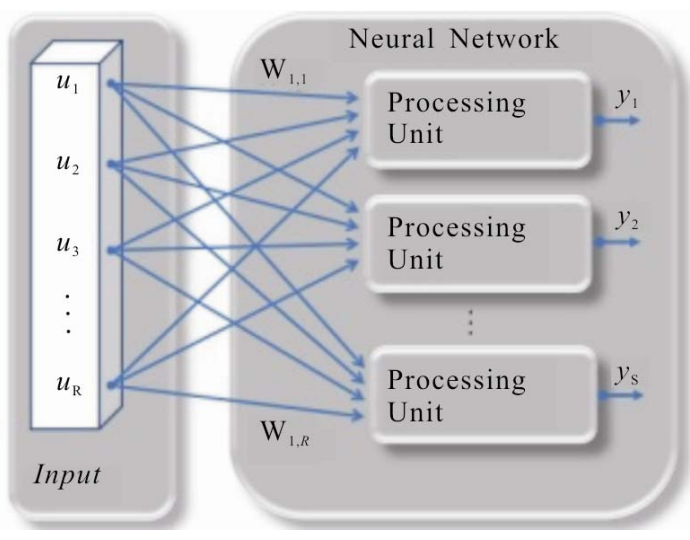

(a)

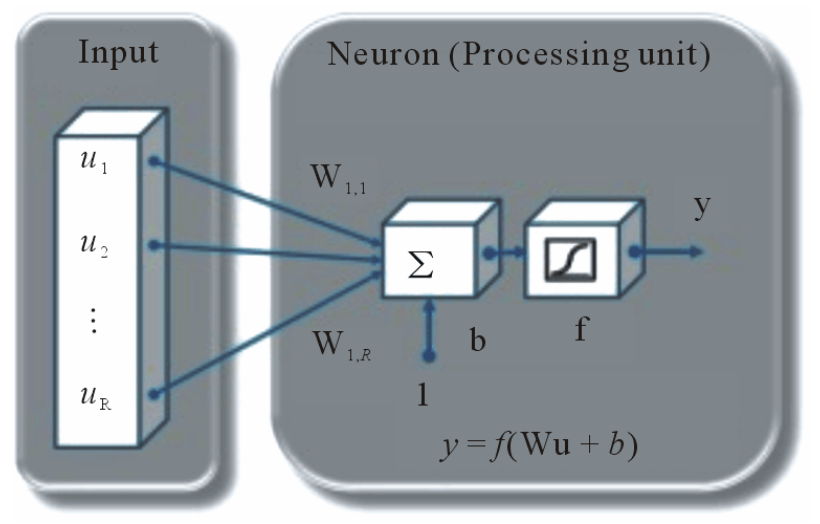

(b)

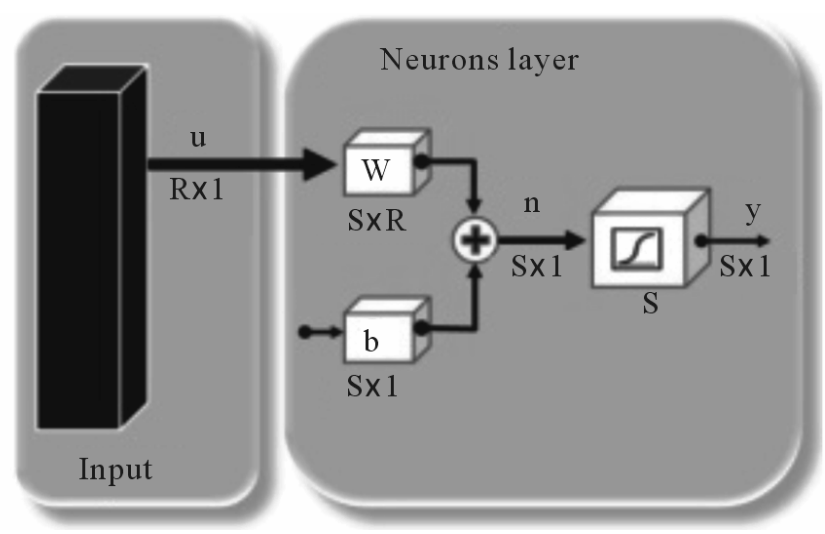

(c)

Figure 3. Neural network with a single layer (a), its processing unit (b) and its diagram (c).

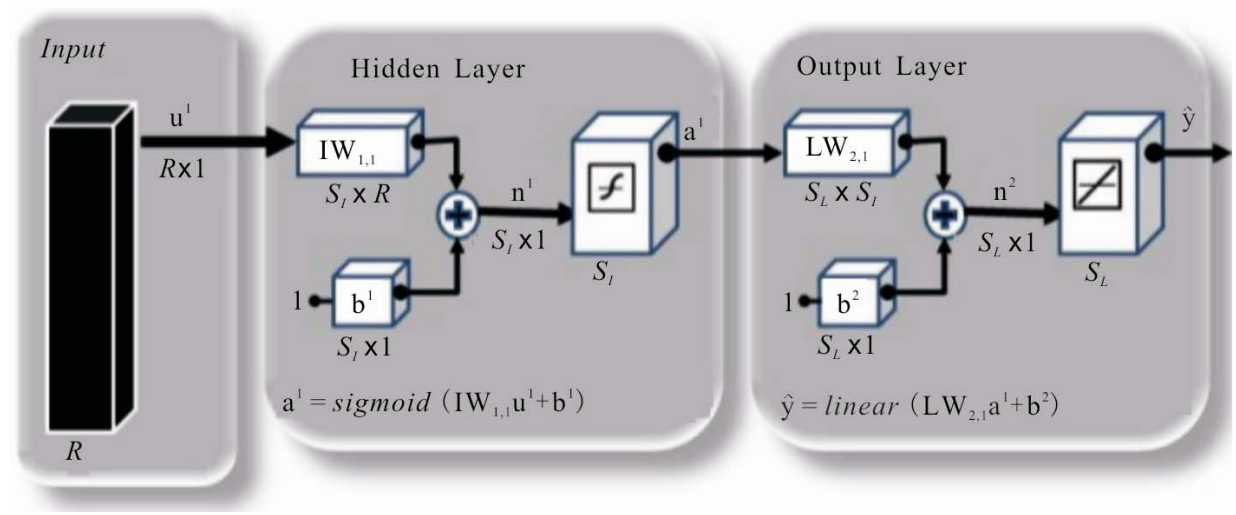

Figure 4. Structure of a network with one hidden layer with sigmoid function and an output layer with linear function (function universal approximator).

Magalhaes [1] presented the development of the machine room transfer function (Machine-Room Transfer Function-MRTF), which simulates the acoustic transmission between the primary source and a receiver in a room, including the spatial distribution of 350 MRTFs (Machine-Room Transfer functions) and a total of 1750 parameters. In order to reduce the number of parameters of the models used to describe the spatial behavior of the acoustic system, an interpolation process on a subset of the identified models was applied. This procedure resulted in a model with 135 parameters, a significant reduction (about 93\%) in the total number of parameters, maintaining a good description of the dominant dynamic of the system, with no degradation in the output signal. The output signal was considered degraded when the Average Euclidean norm [26] of the errors of the estimated output model was greater than $80 \%$ of the Euclidean norm of the output signal (Table 1). This reduction 


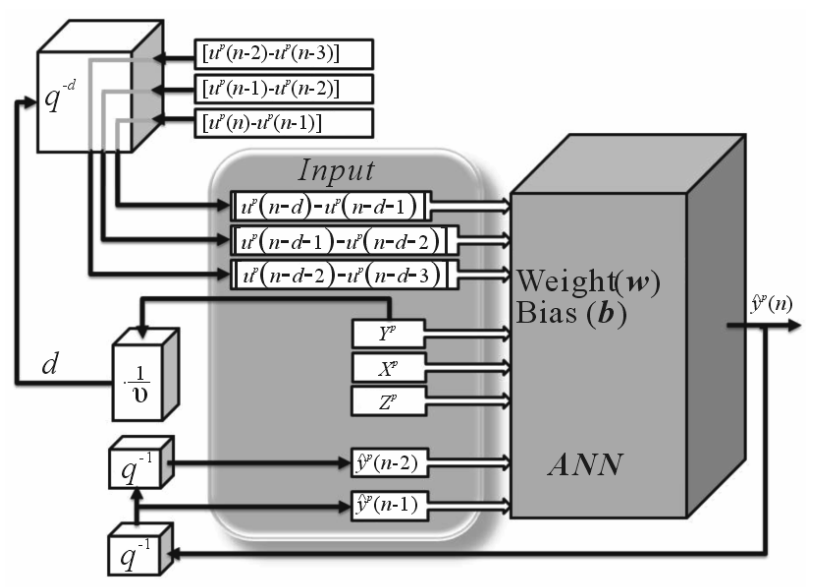

(a)

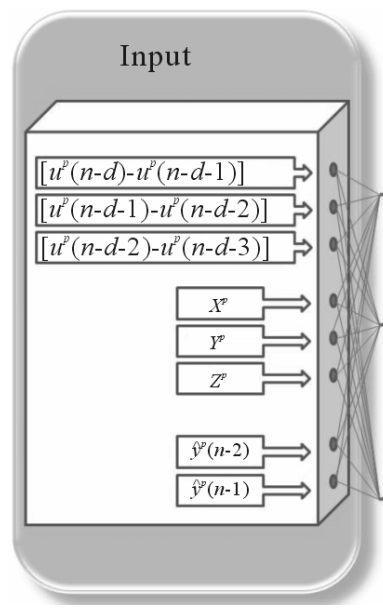

(b)

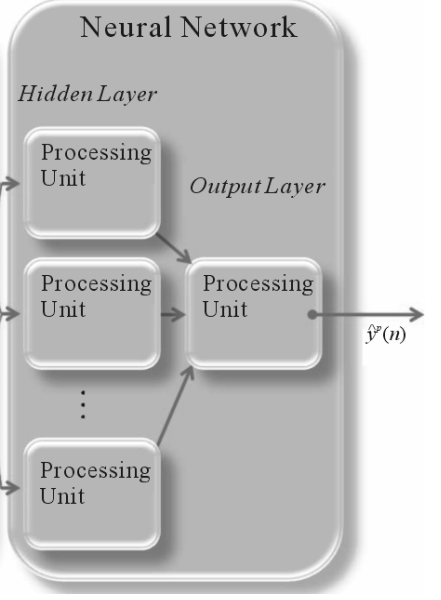

Figure 5. ANN structure (long-range prediction) (a) and details of the internal structure adopted (b).

Table 1. Comparison of the Euclidean norm of the errors.

\begin{tabular}{lcccc}
\hline $\begin{array}{l}\text { Errors Euclidean Norm }\left(\mathbf{P a}^{2}\right): \\
\text { Models: }\end{array}$ & Average & Minimum & Maximum & Number of parameters \\
\hline Euclidean norm of the output signal & 11.8 & 4.9 & 21.6 & - \\
Identified ARX without delay (350 MRTFs) & 7.3 & 3.6 & 15.8 & 1750 \\
Identified ARX with delay (350 MRTFs) & 7.1 & 4.1 & 13.3 & $\mathbf{1 3 5}$ \\
Interpolated ARX with delay (27 MRTFs) & $\mathbf{9 . 3}$ & $\mathbf{4 . 6}$ & $\mathbf{2 0 . 8}$ & 90 \\
Interpolated ARX with delay (18 MRTFs) & 9.8 & 4.6 & 22.0 & 35 \\
Interpolated ARX with delay (9 MRTFs) & 10.8 & 5.0 & 19.9 & 20 \\
Interpolated ARX with delay (4 MRTFs) & 11.0 & 5.0 & 19.9 & $\mathbf{8 0}$ \\
ANN with delay & $\mathbf{9 . 3}$ & $\mathbf{4 . 9}$ & $\mathbf{1 6 . 7}$ & $\mathbf{8 0}$ \\
\hline
\end{tabular}

allows future implementation of this model structure in control systems in real-time.

For the purpose of comparing the present structure with that obtained in the work of Magalhaes [1], the maximum number of parameters for the current network was established to be less than 135, the number of parameters adopted by Magalhães and coworkers. This assumption is supposed to produce no hammering in the quality obtained for the proposed model, nor in the comparison outcome with the previous model. The total number of parameters in an ANN (Figure 4) with one hidden layer (intermediate) and one neuron in the output layer is given by:

$$
N_{p}=R \cdot S_{I}+2 \cdot S_{I}+1
$$

where $R$ is the number of network inputs and $S_{I}$ is the number of neurons of the hidden layer. In this work, $N_{p} \cong 80$ was adopted in order to keep the resulting number of parameters less than 135, as stated earlier. With the input layer defined according to the Figure 5 $(R=8)$ and using Equation (1), the number of neurons in hidden layer was equal to 8 .

\subsection{Procedure for Parameter Estimation: Training of the Recurrent Network}

Once the network topology is characterized, it is necessary to establish the training procedure, which was formulated through the optimization procedure shown in Figure 6, where $e(n)$ represents the simulation error.

Thus, the training of the network was formulated as a general problem of nonlinear optimization with constraints [27,28]:

$$
\begin{aligned}
& \min _{x} f(x) \\
& \text { subject to } \\
& G_{i}(x)=0, i=1, \cdots, m_{e} \\
& G_{i}(x) \leq 0, i=m_{e}+1, \cdots, m
\end{aligned}
$$

where $x$ is the vector of parameters of length $n, f(x)$ is the objective function, which gives a scalar value, and the vector function $G(x)$ gives a vector of length $m$ 


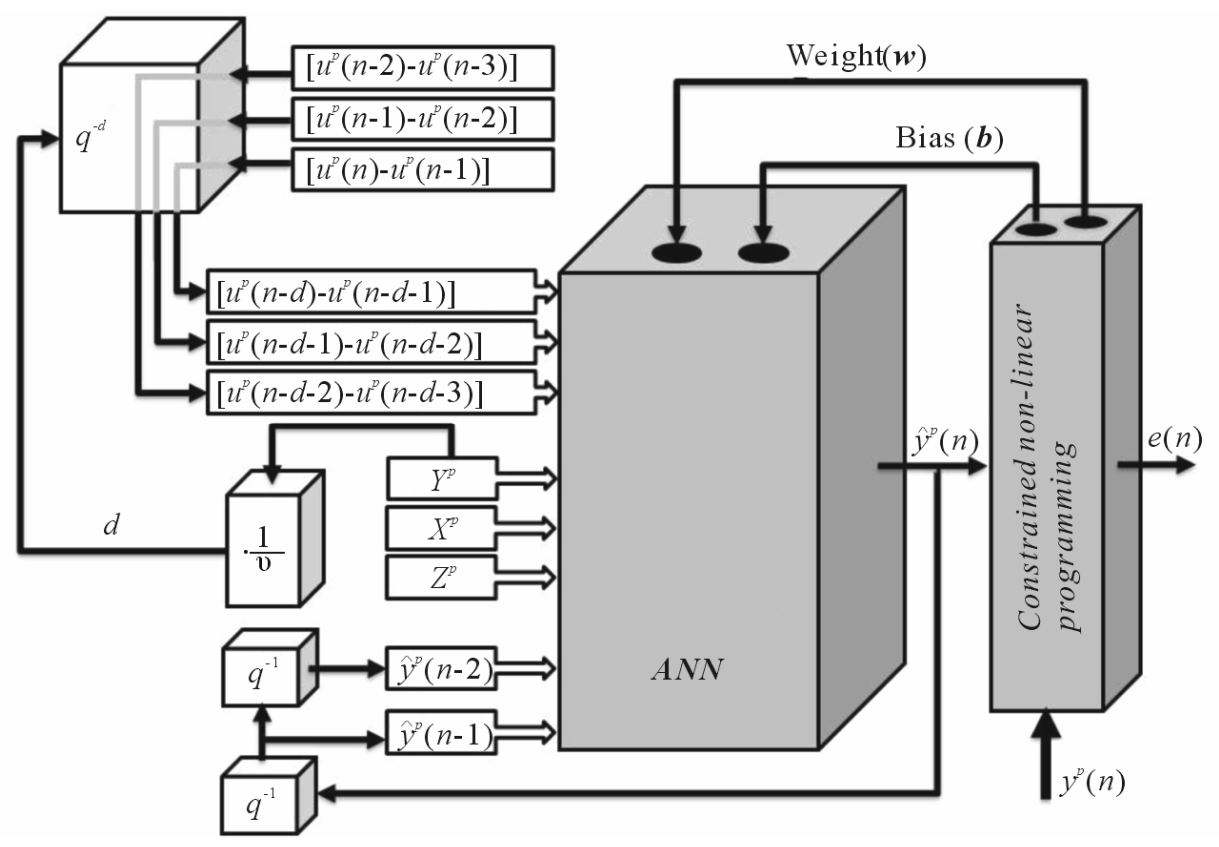

Figure 6. Recurrent ANN training (long-range prediction).

containing the values of equalities and inequalities constraints which are evaluated at $x$. Constraints are usually used to achieve certain desired properties for the network or to restrict the search region to avoid convergence problems. For both the topology and the experimental data used in our ANN model, no constraints were necessary because the optimization algorithm behaved smoothly in most of the runs.

The solutions of the Khun-Tucker (KT) [29], equations are the basis for many nonlinear programming algorithms. The methods that use these algorithms are commonly referred to as Sequential Quadratic Programming (SQP). SQP methods, described in [30-32] works, offer a good method for solving the problem of ANN dynamic optimization. A SQP overview can be found in [33].

The training was performed using MATLAB ${ }^{\circledR}$. The availability of a good initial estimate is an important requisite for success in solving these problems. In this case, an initial guess was used to train the model according to the scheme shown in Figure 7 which consists of an identification procedure based on one-step ahead prediction. A set of patterns was used made up of pairs that were collected in the experiment $\left(\left\{\boldsymbol{u}^{p}, \boldsymbol{y}^{p}\right\}, p=1,2, \cdots, 350\right)$ which defines the input vector for each time $n$, according to Equation (3). A network able to do one-step ahead prediction is not the final network of interest rather a dynamic model able to perform long-range prediction is desirable (Figure 6). However, the one-step ahead prediction network (Figure 7) provides a good initial estimate for the optimization procedure shown in Figure 6. Thus, the parameters (weights and bias) obtained through the one-step ahead optimization procedure for the ANN training (Figure 7) are used as initial condition for the optimization procedure defined for the recurrent ANN training (long-range prediction) (Figure 6). In the optimization procedure used here (Figures 6 and 7), the objective function, which is used in the networks training, is the average of the square root of the sum of square errors (difference between the measured value and the value that was estimated by the model for each output value, which is given by $e(n)$ ), for $n=1, \cdots, 390$.

$$
\operatorname{Input}(n)=\left[\begin{array}{c}
{\left[u^{p}(n-d)-u^{p}(n-d-1)\right]} \\
{\left[u^{p}(n-d-1)-u^{p}(n-d-2)\right]} \\
{\left[u^{p}(n-d-2)-u^{p}(n-d-3)\right]} \\
X^{p} \\
Y^{p} \\
Z^{p} \\
y^{p}(n-2) \\
y^{p}(n-1)
\end{array}\right]_{p=1}
$$

\section{Results and Discussion}

As stated earlier, it is necessary to establish a metric (criterion) to be used in the objective function. This metric can have a decisive influence in the determination of the optimal point as the optimization procedure will seek the parameters that lead the model to the best possible performance in relation to these established criteria. In this article Euclidean distance is used [34], a metric that 


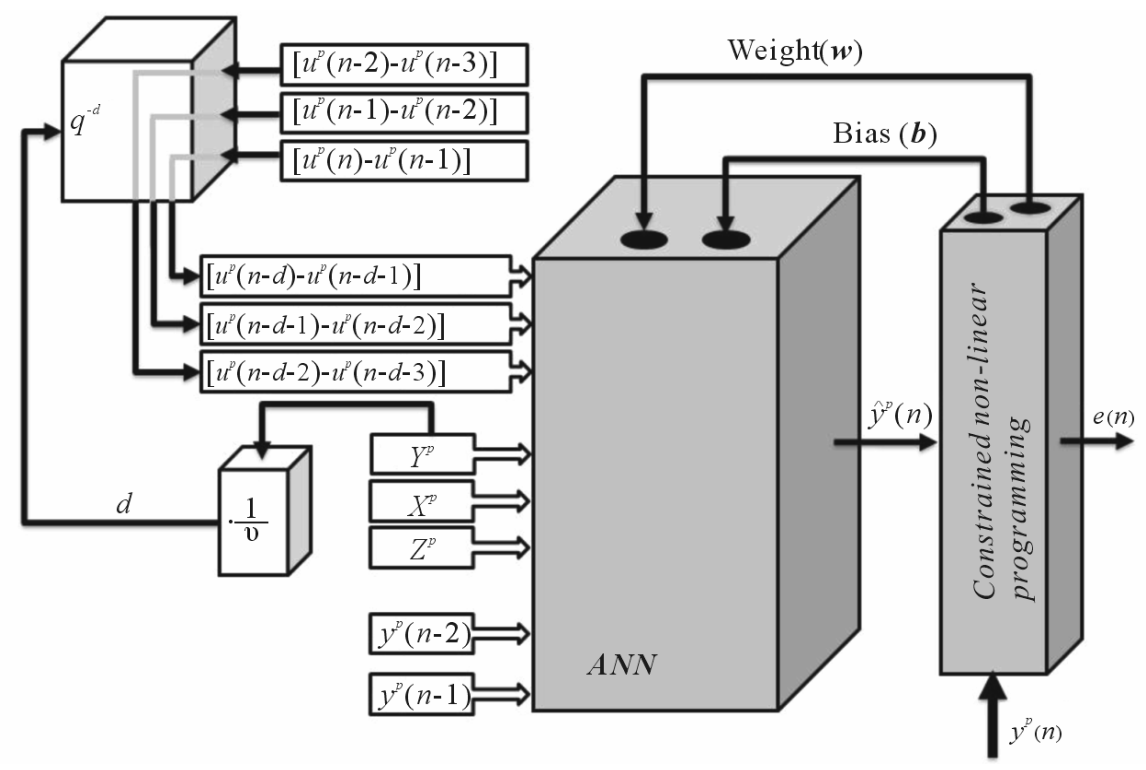

Figure 7. ANN training (one-step ahead prediction).

is commonly used in the treatment of causal events in order to obtain an objective function that gives the distance of functional responses (responses of the models) in relation to its target profile (experimental output). Euclidean distance is also commonly called the error vector norm $(\mathrm{EVN})$ and is given by:

$$
E V N_{s, T D}=\sqrt{\sum_{n=1}^{T P}\left(y_{\operatorname{Mod}(s, n)}-y_{\operatorname{Exp}(s, n)}\right)^{2}}, s=1, \cdots, S P
$$

for the time domain, and by:

$$
\begin{aligned}
& E V N_{s, F D}=\sqrt{\sum_{k=1}^{T P}\left(Y_{\operatorname{Mod}(s, \omega)}-Y_{\operatorname{Exp}(s, \omega)}\right)^{2}}, s=1, \cdots, S P \\
& Y_{\operatorname{Mod}(s, \omega)}=\sum_{n=1}^{T P} y_{\operatorname{Mod}(s, n)} \mathrm{e}^{-j \cdot 2 \cdot \pi \cdot(k-1) \cdot(n-1) / T P}, k=1, \cdots, T P \\
& Y_{\operatorname{Exp}(s, \omega)}=\sum_{n=1}^{T P} y_{\operatorname{Exp}(s, n)} \mathrm{e}^{-j \cdot 2 \cdot \pi \cdot(k-1) \cdot(n-1) / T P}, k=1, \cdots, T P \\
& \omega=h(k)=\frac{2 \cdot \pi \cdot(k-1)}{T P}
\end{aligned}
$$

for the frequency domain, where $S P$ and $T P$ are the numbers of points in space and in time, respectively, $y$ and $Y$ are the outputs in the time and in the frequency domains respectively, the subscripts Mod and Exp refer to the model and to the experimental output respectively, the subscript $s$ defines a specific spatial position, $k$ and $\omega$ are the discrete time and frequency, respectively, the subscripts $T D$ and $F D$ refer to the domains of time and frequency, respectively, and $h$ symbolizes the functional relationship between $\omega$ and $k$.

In the optimization procedure used here (Figures 6 and 7), both metrics established by the objective functions described in Equations (4) and (5) were tested, as well as their combinations, obtaining equivalent models. Here, for the purpose of numerical comparison between the different modeling approaches, Equation (4) was assumed.

The resulting models were used to predict the dynamic and spatial behavior of the system's output signal (acoustic power measured by microphone). From Figures 8-10 the best and the worst results of the models in the estimation of the output signal for plans $Z=1,3$ and 5 can be seen, as well as a qualitative comparison with estimated results with the models that were obtained in the work of Magalhaes [1]. It can be seen that even the worst results with the ANN models provide an adequate dynamic representation of the experimental data, capturing the main trends of the system behavior, although the amplitudes at higher frequencies have a strong attenuation, as can be seen in Figure 11, which shows the average PSD (Power Spectral Density) for 350 mesh points. The ANN model provides a good system dominant dynamic description (PSD peaks), where most of the signal energy is concentrated).

Table 1 compares the result of the model structure proposed in this work with the results obtained by Magalhaes [1]. Based on these results, the following conclusions may be highlighted:

- Smaller errors are obtained when considering the delays in each $X Z$ plane to identify the models in the various grid points collected;

- According to the results of Magalhaes [1], the maximum possible reduction in the mesh for the application of the interpolation was a reduction to 27 MRTFs. As can be seen in Table 1, any reduction superior to this takes the average, minimum or maximum values of the error norm to higher values compared to the 
Worst Adjustments ANN

$\mathrm{Z}=1(0.44 \mathrm{~m}), \mathrm{X}=1(0.44 \mathrm{~m})$ and $\mathrm{Y}=7(0.44 \mathrm{~m})$,

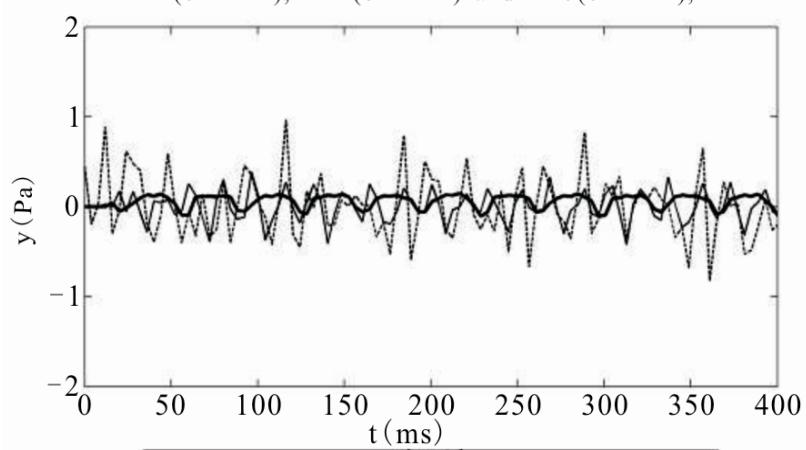

...-...-Measured output $y(n)$

- Estimated output $\mathrm{y}_{\mathrm{p}}(\mathrm{n})$-ARX identified models

- Estimated output $y_{p}(n)-A N N$ models
Best Adjustments ANN

$Z=1(0.44 \mathrm{~m}), X=5(0.44 \mathrm{~m})$ and $Y=2(0.44 \mathrm{~m})$,

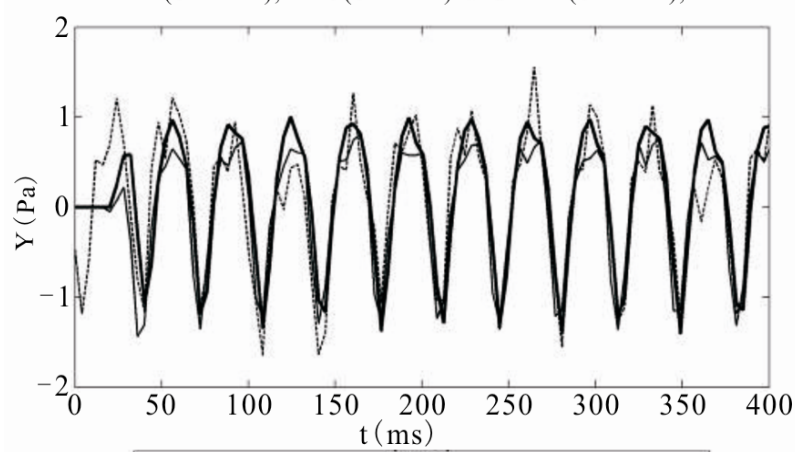

........-Measured output $\mathrm{y}(\mathrm{n})$

- Estimated output $y_{p}(n)-A R X$ identified models

- Estimated output $\mathrm{y}_{\mathrm{p}}(\mathrm{n})$-ANN models

Figure 8. Best and worst adjustments for plane $Z=1 \times(0.44 \mathrm{~m})$ : time response of ARX identified models and ANN models.

Worst Adjustments ANN

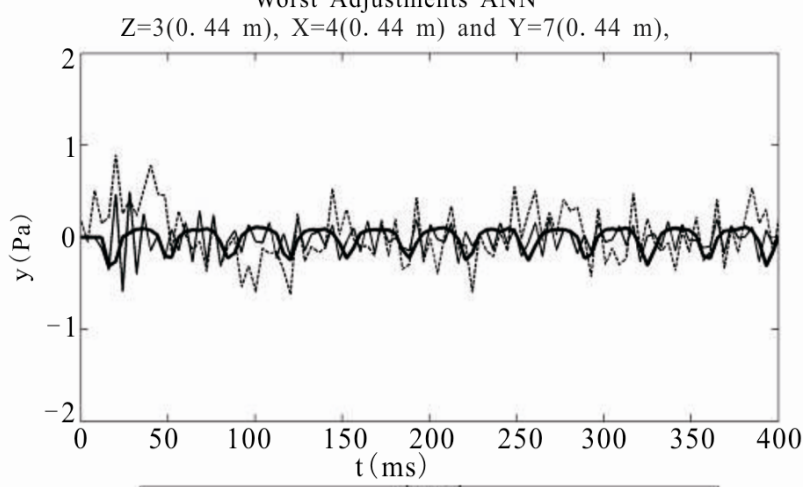

......... Measured output y $(\mathrm{n})$

- Estimated output $y_{p}(n)-A R X$ identified models

- Estimated output $\mathrm{y}_{\mathrm{p}}(\mathrm{n})-\mathrm{ANN}$ models
Best Adjustments ANN $\mathrm{Z}=3(0.44 \mathrm{~m}), \mathrm{X}=2(0.44 \mathrm{~m})$ and $\mathrm{Y}=2(0.44 \mathrm{~m})$,

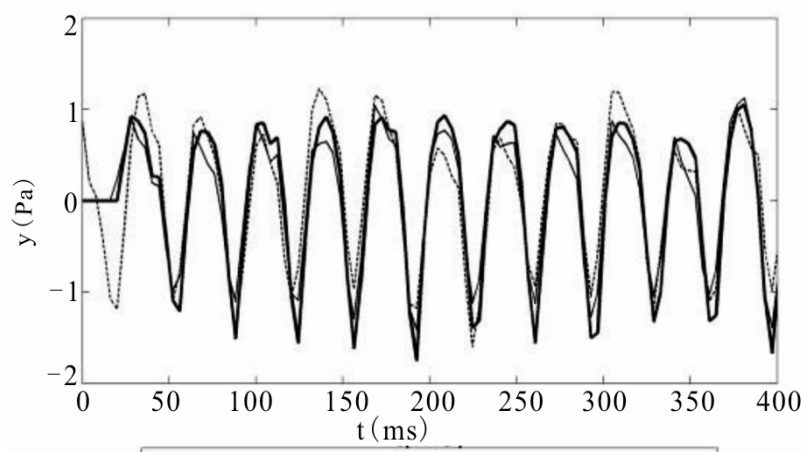

-...-...-Measured output $\mathrm{y}(\mathrm{n})$

- Estimated output $\mathrm{y}_{\mathrm{p}}(\mathrm{n})$-ARX identified models

- Estimated output $\mathrm{y}_{\mathrm{p}}(\mathrm{n})$-ANN models

Figure 9. Best and worst adjustments for plane $Z=3 \times(0.44 \mathrm{~m})$ : time response of ARX identified models and ANN models.
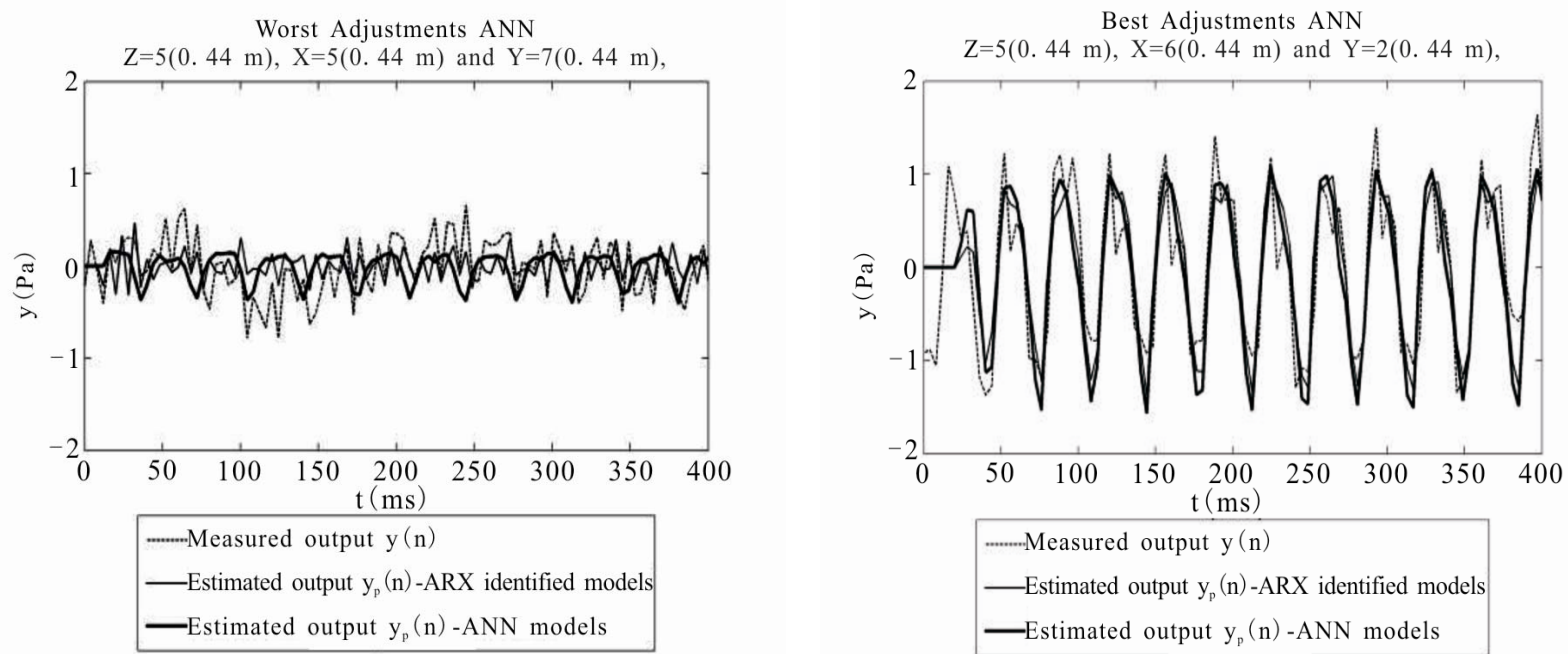

Figure 10. Best and worst adjustments for plane $Z=5 \times(0.44 \mathrm{~m})$ : time response of ARX identified models and ANN models. 


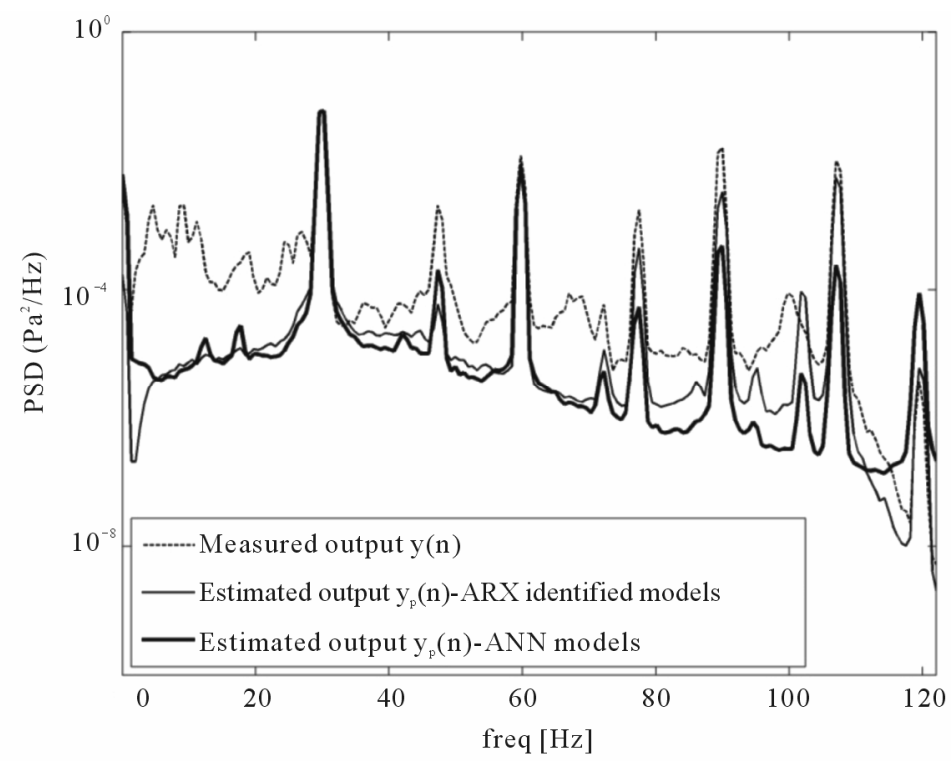

Figure 11. Average PSD for the output.

respective Euclidean norm values of the experimental signal (the objective function, which is used in the networks training, is the average of the square root of the sum of square errors);

- The ANN model performed equivalent to the ARX model (27 MRTFs, 135 parameters), with the advantage that it used fewer parameters ( 80 parameters).

\section{Conclusion}

This paper presented the development of an Artificial Neural Network (ANN) to describe the vibrate-acoustic transmission between a primary source of noise and a receiver in a room. The obtained dynamic ANN model captured the main dynamics of the system and performed equivalent to the performance presented by an ARXinterpolated model [1]; however, with fewer parameters. Considering that these two model structures have a different number of parameters, the dynamic ANN model demonstrated better adherence to the experimental data, providing the smallest variance errors in the estimate of the system output (Table 1). It is possible to further improve the performance of the dynamic ANN model, although this requires a change in the proposed structure for this model, such as increasing the number of neurons in the hidden layer. Strategies like this, however, would increase the number of model parameters, requiring greater computational effort to simulate the outputs of the system. We need models that have the ability to simulate (qualitatively and quantitatively) the dynamics of vibrate-acoustic systems because the ultimate goal of this research project is the development of three-dimensional models for use in the construction of systems to Active Noise Control (ANC). In this case, we also need models with low computational cost, and therefore models with increased number of neurons would not satisfy this requirement.

\section{Acknowledgements}

The authors acknowledge CAPES and CNPq (Brazilian federal research agencies) for their financial support.

\section{REFERENCES}

[1] R. S. Magalhaes, C. H. O. Fontes, L. A. L. Almeida, M. Embirucu and J. M. C. Santos, "A Model for ThreeDimensional Simulation of Acoustic Emissions from Rotating Machine Vibration," Journal of the Acoustical Society of America, Vol. 127, No. 6, 2010, pp. 3569-3576. doi:10.1121/1.3425736

[2] R. S. Magalhaes, C. O. H. Fontes, L. A. L. Almeida, J. M. C. Santos and M. Embirucu, "A Model for Three-Dimensional Simulation of Acoustic Emissions from Rotating Machine Vibration," Composites 2009, 2nd ECCOMAS Thematic Conference on the Mechanical Response of Composites, London, 1-3 April 2009.

[3] B. O. Kachanov, "Symmetric Laplace Transform and Its Application to Parametric Identification of Linear Systems," Automation and Remote Control, Vol. 70, No. 8, 2009, pp. 1309-1316. doi:10.1134/S0005117909080049

[4] E. L. Hines, E. Llobet and J. W. Gardner, "Electronic Noses: A Review of Signal Processing Techniques," IEEE Proceedings-Circuits Devices and Systems, Vol. 146, No. 6, 1999, pp. 297-310. doi:10.1049/ip-cds:19990670

[5] V. M. Becerra, F. R. Garces, S. J. Nasuto and W. Holderbaum, "An Efficient Parameterization of Dynamic Neural Networks for Nonlinear System Identification," IEEE Transactions on Neural Networks, Vol. 16, No. 4, 2005, pp. 983-988. doi:10.1109/TNN.2005.849844 
[6] J. Madar, J. Abonyi and F. Szeifert, "Genetic Programming for the Identification of Nonlinear Input-Output Models," Industrial \& Engineering Chemistry Research, Vol. 44, No. 9, 2005, pp. 3178-3186. doi:10.1021/ie049626e

[7] V. Prasad and B. W. Bequette, "Nonlinear System Identification and Model Reduction Using Artificial Neural Networks," Computers and Chemical Engineering, Vol. 27, No. 12, 2003, pp. 1741-1754. doi:10.1016/S0098-1354(03)00137-6

[8] S. M. Siniscalchi and C.-H. Lee, "A Study on Integrating Acoustic-Phonetic Information into Lattice Rescoring for Automatic Speech Recognition," Speech Communication, Vol. 51, No. 11, 2009, pp. 1139-1153. doi:10.1016/j.specom.2009.05.004

[9] L. Yu and J. Kang, "Modeling Subjective Evaluation of Soundscape Quality in Urban Open Spaces: An Artificial Neural Network Approach," Journal of the Acoustical Society of America, Vol. 126, No. 3, 2009, pp. 1163-1174. doi:10.1121/1.3183377

[10] A. Dariouchy, E. Aassif, G. Maze, D. Decultot and A. Moudden, "Prediction of the Acoustic Form Function by Neural Network Techniques for Immersed Tubes," Journal of the Acoustical Society of America, Vol. 124, No. 2, 2008, pp. 1018-1025. doi:10.1121/1.2945164

[11] A. Saxena and A. Saad, "Evolving an Artificial Neural Network Classifier for Condition Monitoring of Rotating Mechanical Systems," Applied Soft Computing, Vol. 7, No. 1, 2007, pp. 441-454. doi:10.1016/j.asoc.2005.10.001

[12] A. C. Tsoi and A. D. Back, "Locally Recurrent Globally Feedforward Networks-A Critical-Review of Architectures," IEEE Transactions on Neural Networks, Vol. 5, No. 2, 1994, pp. 229-239. doi:10.1109/72.279187

[13] A. U. Levin and K. S. Narendra, "Control Of Nonlinear Dynamic-Systems Using Neural Networks - Controllability and Stabilization," IEEE Transactions on Neural Networks, Vol. 4, No. 2, 1993, pp. 192-206. doi:10.1109/72.207608

[14] A. U. Levin and K. S. Narendra, "Contro of Nonlinear Dynamical Systems Using Neural Networks. II. Observability, Identification, and Control," IEEE Transactions on Neural Networks, Vol. 7, No. 1, 1996, pp. 30-42. doi:10.1109/72.478390

[15] R. T. Bambang, "Adjoint EKF Learning in Recurrent Neural Networks for Nonlinear Active Noise Control," Applied Soft Computing, Vol. 8, No. 4, 2008, pp. 1498-1504. doi:10.1016/i.asoc.2007.10.017

[16] C.-Y. Chang and F.-B. Luoh, "Enhancement of Active Noise Control Using Neural-Based Filtered-X Algorithm," Journal of Sound and Vibration, Vol. 305, No. 1-2, 2007, pp. 348-356. doi:10.1016/j.jsv.2007.04.007

[17] Q.-Z. Zhang, W.-S. Gan and Y. Zhou, "Adaptive Recurrent Fuzzy Neural Networks for Active Noise Control," Journal of Sound and Vibration, Vol. 296, No. 4-5, 2006, pp. 935-948. doi:10.1016/j.jsv.2006.03.020

[18] M. Bouchard, "New Recursive-Least-Squares Algorithms for Nonlinear Active Control of Sound and Vibration Using Neural Networks," IEEE Transactions on Neural Networks, Vol. 12, No. 1, 2001, pp. 135-147. doi: $10.1109 / 72.896802$

[19] F. Rosenblatt, "The Perceptron-A Probabilistic Model for Information-Storage and Organization in the Brain," Psychological Review, Vol. 65, No. 6, 1958, pp. 386-408. doi: $10.1037 / \mathrm{h} 0042519$

[20] E. Masson and Y. J. Wang, "Introduction to Computation and Learning in Artificial Neural Networks," European Journal of Operational Research, Vol. 47, No. 1, 1990, pp. 1-28. doi:10.1016/0377-2217(90)90085-P

[21] M. Nikravesh, A. E. Farell and T. G. Stanford, "Model Identification of Nonlinear Time Variant Processes via Artificial Neural Network," Computers \& Chemical Engineering, Vol. 20, No. 11, 1996, pp. 1277-1290. doi:10.1016/0098-1354(95)00245-6

[22] J. X. Zhan and M. Ishida, "The Multi-Step Predictive Control of Nonlinear SISO Processes with a Neural Model Predictive Control (NMPC) Method," Computers \& Chemical Engineering, Vol. 21, No. 2, 1997, pp. 201-210. doi:10.1016/0098-1354(95)00257-X

[23] K. Narendra and K. Parthasarathy, "Identification and Control of Dynamical Systems Using Neural Networks," IEEE Transactions on Neural Networks, Vol. 1, No. 1, 1990, pp. 4-27. doi:10.1109/72.80202

[24] A. J. Meade and H. C. Sonneborn, "Numerical Solution of a Calculus of Variations Problem Using the Feedforward Neural Network Architecture," Advances in Engineering Software, Vol. 27, No. 3, 1996, pp. 213-225. doi:10.1016/S0965-9978(96)00029-4

[25] R. R. Selmic and F. L. Lewis, "Neural-Network Approximation of Piecewise Continuous Functions: Application to Friction Compensation," IEEE Transactions on Neural Networks, Vol. 13, No. 3, 2002, pp. 745-751. doi:10.1109/TNN.2002.1000141

[26] N. Takagi and S. Kuwahara, "A VLSI Algorithm for Computing the Euclidean Norm of a 3D Vector," IEEE Transactions on Computers, Vol. 49, No. 10, 2000, pp. 1074-1082. doi:10.1109/12.888043

[27] L. T. Biegler and I. E. Grossmann, "Restrospective on Optimization," Computers and Chemical Engineering, Vol. 28, No. 8, 2004, pp. 1169-1192. doi:10.1016/j.compchemeng.2003.11.003

[28] P. E. Gill, W. Murray and M. A. Saunders, "A Practical Anti-Cycling Procedure for Linearly Constrained Optimization," Mathematical Programming, Vol. 45, No. 3, 1989, pp. 437-474. doi:10.1007/BF01589114

[29] O. A. Brezhneva, A. A. Tret'yakov and S. E. Wright, “A Simple and Elementary Proof of the Karush-Kuhn-Tucker Theorem for Inequality-Constrained Optimization," Optimization Letters, Vol. 3, No. 1, 2009, pp. 7-10. doi:10.1007/s11590-008-0096-3

[30] M. C. Biggs, "Convergence of Some Constrained Minimization Algorithms Based on Recursive Quadratic Programming," Journal of the Institute of Mathematics and Its Applications, Vol. 21, No. 1, 1978, pp. 67-81. doi:10.1093/imamat/21.1.67

[31] S. P. Han, "Globally Convergent Method for Nonlinear-Programming," Journal of Optimization Theory and Applications, Vol. 22, No. 3, 1977, pp. 297-309. 
doi:10.1007/BF00932858

[32] R. P. Ge and M. J. D. Powell, "The Convergence of Variable-Metric Matrices in Unconstrained Optimization," Mathematical Programming, Vol. 27, No. 2, 1983, pp. 123-143. doi:10.1007/BF02591941

[33] W. Hock and K. Schittkowski, "A Comparative Performance Evaluation of 27 Non-Linear Programming Codes,"
Computing, Vol. 30, No. 4, 1983, pp. 335-358. doi:10.1007/BF02242139

[34] P. E. Danielsson, "Euclidean Distance Mapping," Computer Graphics and Image Processing, Vol. 14, No. 3, 1980, pp. 227-248. doi:10.1016/0146-664X(80)90054-4 